\section{International Scientific Journal Theoretical \& Applied Science}

Nikolay Vasilevich Penshin candidat of economic sciences, Head of the Department "Organization of transportation and road safety" Tambov state technical University, Russia avtobd@mail.ru

Victor Yurievich Ivlev Undergraduate, Mechanical engineer Aor Nekrasovskoe RTP, city of Rasskazovo, Russia victor.pshenihniy_7@mail.ru

SECTION 27. Transport.

\title{
METHODS OF CERTIFICATION TESTS VEHICLES FOR COMPLIANCE WITH ACTIVE, PASSIVE, POST-ACCIDENT AND ENVIRONMENTAL SAFETY
}

Abstract: Certification tests of motor transport is complicated, time-consuming and expensive process that involves a huge number of modern control equipment, highly qualified specialists and, in addition, special stands.

Key words: Car, certification, traffic safety

Language: Russian

Citation: Penshin NV, Ivlev VY (2015) METHODS OF CERTIFICATION TESTS VEHICLES FOR COMPLIANCE WITH ACTIVE, PASSIVE, POST-ACCIDENT AND ENVIRONMENTAL SAFETY. ISJ Theoretical \& Applied Science 12 (32): 103-107.

Soi: http://s-o-i.org/1.1/TAS-12-32-20 Doi: crossef http://dx.doi.org/10.15863/TAS.2015.12.32.20

\section{УДК 502.7}

\section{МЕТОДЫ СЕРТИФИКАЦИОННЫХ ИСПЫТАНИЙ АВТОМОБИЛЕЙ НА СООТВЕТСТВИЕ АКТИВНОЙ, ПАССИВНОЙ, ПОСЛЕАВАРИЙНОЙ И ЭКОЛОГИЧЕСКОЙ БЕЗОПАСНОСТИ}

Аннотация: Сертификационные испытания автомобильного транспорта - это достаточно сложный, длительный по времени, а также дорогостоящий процесс, в котором задействовано огромное количество современной контрольной аппаратуры, высококвалифицированных специалистов и, кроме того, специильных стендов.

Ключевые слова: Автомобиль, сертификащия, безопасность дорожного движения.

Для развития экономики каждой страны важная роль принадлежит автомобильному транспорту.

При сертификации автомобильной техники проводят такие основные виды испытаний, как [2, c.13]:

- соответствие характеристик автомобиля пассивной, активной, а также послеаварийной безопасности;

- испытание на топливную и динамичную экономичность;

- испытание на экологическую безопасность;

- испытание автомобилей на шумность.

Активная безопасность автомобильного транспорта представляет собой свойство, благодаря которому он способен предотвратить дорожно-транспортное происшествие, т.е. снизить вероятность его возникновения.
Активная безопасность транспортного средства (ТС) состоит из [8, с.38]:

- тормозных свойств;

- управляемости компоновочных параметров автомобиля (весовые и габаритные);

- тяговой динамичности;

- информативности;

- устойчивости;

- оборудования рабочего места водителя, а также его соответствия требованиям эргономики;

- обзорности дороги (зеркала заднего вида, ветровое и боковые стекла);

- надежности транспортных средств, их элементов оборудования и комплектующих, которые влияют на вероятность возникновения дорожно-транспортного происшествия (ДТП).

Наиважнейшие факторы активной безопасности автомобильного транспорта проявляются в (рис.1):

- защищенности его от шума и вибраций; 
- удобстве размещения водителя;

- характеристике микроклимата;

- обзорности и информативности панели приборов;
- доступности и удобстве использования органов управления и т.д. [8, с. 54]

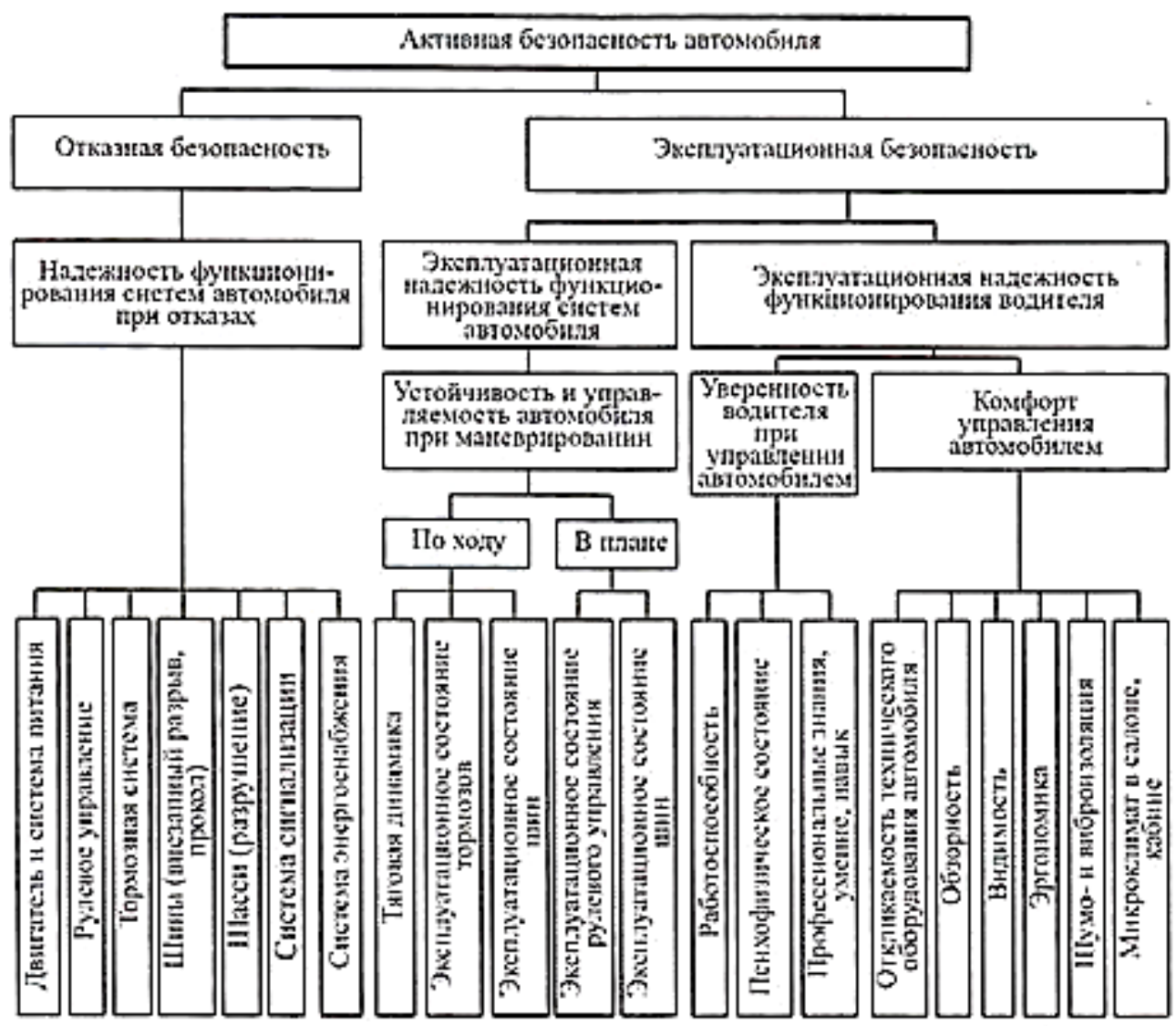

Рисунок 1 - Структурная схема активной безопасности автомобиля.

На вероятность возникновения дорожнотранспортого происшествия влияют такие свойства как [7, с.13]:

- тормозная динамичность;

- устойчивость;

- управляемость.

В основе технологии испытания тормозных свойств лежат положения теории автомобиля, которые устанавливают связь сил, которые действуют в процессе движения на него и моментов с параметрами конструкции. Основываясь на эти положения, можно установить показатель тормозных свойств результативность (эффективность) торможения, а параметрами же его количественной оценки выбирают установившееся замедление, тормозной путь, тормозную силу, время срабатывания тормозов и время до полной остановки.

Результативность торможения следует оценивать при:

- использовании разных тормозных систем, которые стали в современных автомобилях

ISPC Applied technologies,

Scranton, USA обязательными (запасной,

рабочей, вспомогательной, стояночной);

- разных состояниях тормозных механизмов (мокрых, а также нагретых);

- при типовых условиях различного воздействия внешних факторов.

Устанавливают три главных типа испытаний: 0, 1 и 2.

При испытаниях типа «0» измеряют результативность торможения при наличии холодных тормозных механизмах, в таких случаях температура наружных поверхностей тормозных дисков или же тормозных барабанов колес сохранена в пределах $50-100^{\circ} \mathrm{C}$.

При испытании типа «1» непосредственно измеряют строго регламентированным образом результативность торможения после нагрева тормозов.

В испытаниях же типа «2» оценивают результативность тормозов автомобиля после движения, которое определенным образом ограничено, с торможением на затяжных спусках [3, c.17]. 
Оценка технологии тормозных испытаний включает [10]:

- эффективность и восстанавливаемость мокрых тормозных механизмов;

- термонагруженность и эффективность охлаждения тормозных механизмов;

- эффективность рабочей, стояночной, запасной, вспомогательной тормозных систем;

- устойчивость при торможении;

- совместимость тормозных свойств прицепа и, кроме того, тягача у автопоезда;

- время срабатывания тормозного привода;

- эффективность инерционности тормозной системы отдельного прицепа и, кроме того, в составе автопоезда.

Управляемость представляет собой свойство подчинения автомобиля действиям водителя в целях сохранности или же изменения вектора, а также величины скорости движения и, кроме того, ориентации продольной оси автомобиля.
Устойчивость представляет собой свойство сохранности автомобиля заданных направлений движения (траекторная устойчивость), ориентации продольной оси (курсовая устойчивость) и, кроме того, вертикальной оси (устойчивость против опрокидывания).

Пассивная безопасность автомобиля представляет собой свойство автомобиля снижать тяжесть последствий дорожно-транспортных происшествий.

В отличие же от активной, пассивная безопасность характеризует собой способность конструкции ослабить или предотвратить травмирование пассажиров и водителя в случившемся дорожно-транспортном происшествии.

Структурная схема элементов пассивной безопасности автомобиля представлена на рис. 2 $[8, \mathrm{c.138}]$.

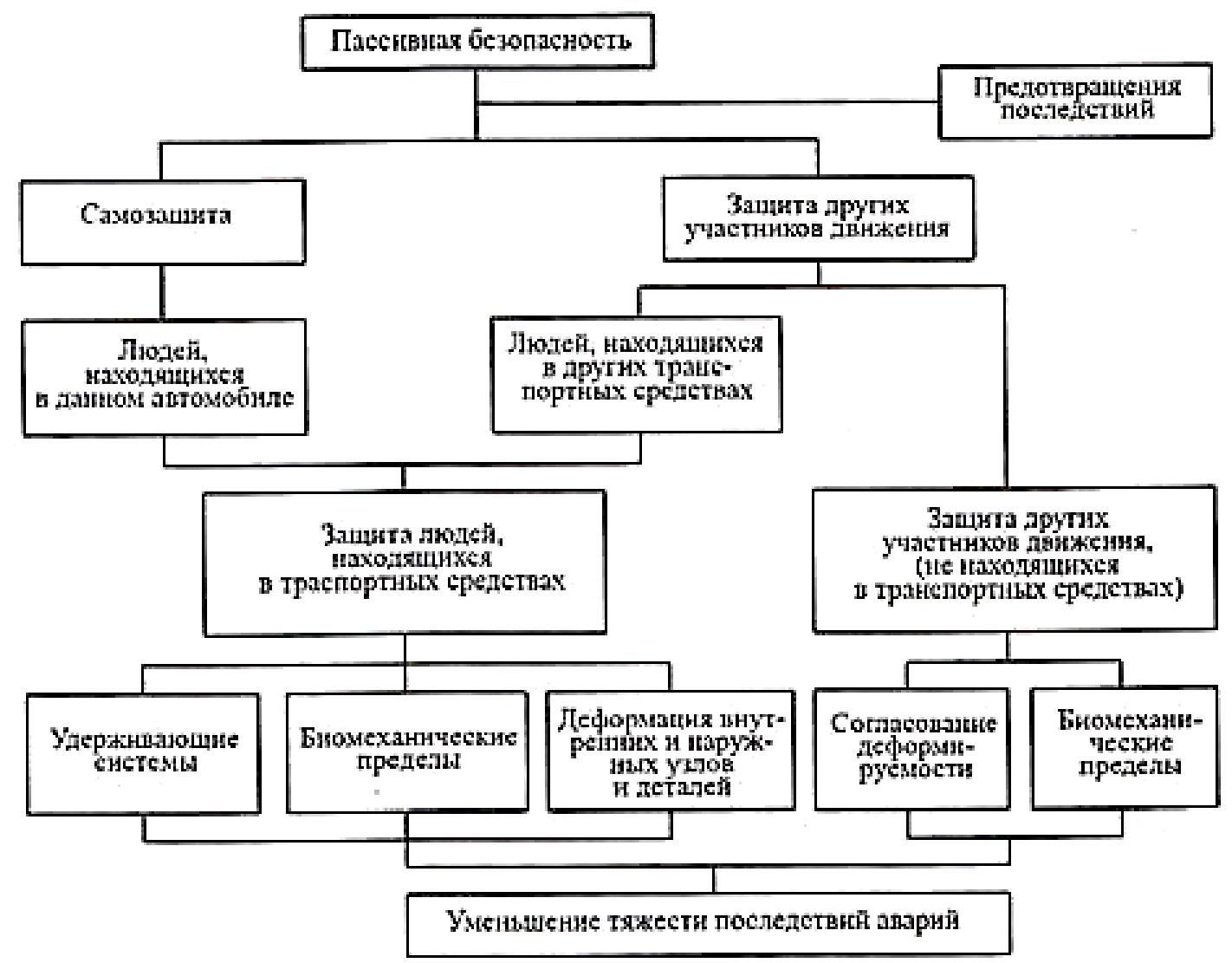

Рисунок 2 - Структурная схема пассивной безопасности автомобиля.

Статистика, а также исследования ДТП и, кроме того, их последствий показывают [9]:

- различную степень опасности поврежденности отдельных элементов в конструкции;

- существенные различия по тяжести последствий видов ДТП.
В современных условиях выработано более чем двадцать требований для обеспечения конкретного уровня безопасности к конструкции автомобильной техники, среди которых выделяют требования безопасности при более тяжелом виде ДТП - лобовом или же фронтальном ударе. 
Также учитывают то обстоятельство, что 80\% всех фронтальных столкновений, в соответствии со статистическими данными, с неподвижными или же движущимися объектами составляют всегда прямые центральные удары, количество погибающих пассажиров, а также водителей при которых достигает более чем 40\% общего количества жертв ДТП всех видов [10].

На рисунке 3 показана условно динамика изменения уровней безопасности комплекса «Водитель-Автомобиль-Дорога-Среда» в разных фазах ДТП [8, с.145].

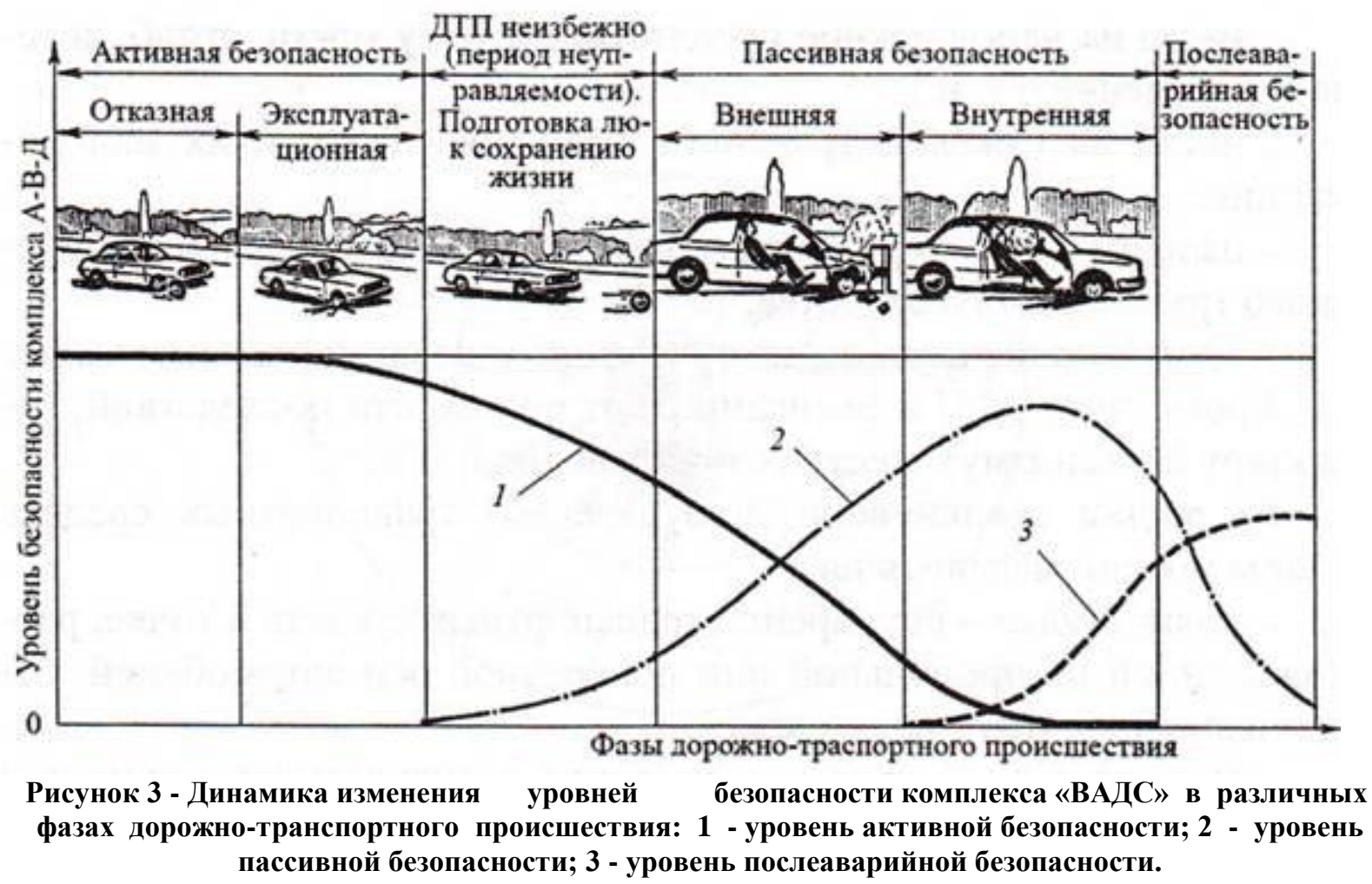

Послеаварийная безопасность автомобиля представляет собой свойство автомобиля снижать тяжесть последствий ДТП после его остановки, называемая конечной фазой ДТП. Данное свойство характеризует возможностью достаточно быстрой ликвидации последствий происшествия и, кроме того, предотвращением возникновения новых аварийных ситуаций.

Пассивная и послеаварийная безопасность ТC тесно взаимосвязаны и, как правило, они рассматриваются совместно.

В современных условиях технология испытаний пассивной безопасности построена на натурных экспериментах полнокомплектных образцов автомобилей, содержание которой при фронтальном столкновении излагается в Правилах №33 ЕЭК ООН - «Единообразные предписания, касающиеся официального утверждения транспортных средств в отношении поведения их конструкции в случае лобового столкновения».
Одновременно оценивается и послеаварийная безопасность автомобиля, по следующим правилам [4, с.21]:

- от действия удара боковые двери открываться не должны, а необходимо обеспечение возможности открытия после удара не применения инструменты количества дверей в целях эвакуации находящихся в автомобиле;

- никакой жесткий элемент в салоне после испытаний не должен представлять опасность серьезного ранения пассажиров или же водителя.

Экологическая безопасность автомобиля представляет собой свойство автомобиля, которое позволяет уменьшать вред, который при нормальной его эксплуатации наносится участникам движения и, кроме того, окружающей среде [8, с.204].

Таким образом, виды безопасности, которые были описаны выше, рассматриваются, как правило, в изоляции друг от друга, но это делают в целях простоты их изучения. 
\begin{tabular}{l|lrl|l|ll} 
& ISRA (India) & $=\mathbf{1 . 3 4 4}$ & SIS (USA) & $=\mathbf{0 . 9 1 2}$ & ICV (Poland) & $=\mathbf{6 . 6 3 0}$ \\
Impact Factor: & ISI (Dubai, UAE) $=\mathbf{0 . 8 2 9}$ & PUHIL (Russia) $=\mathbf{0 . 1 7 9}$ & PIF (India) & $=\mathbf{1 . 9 4 0}$ \\
& GIF (Australia) & $\mathbf{0 . 5 6 4}$ & ESJI (KZ) & $=\mathbf{1 . 0 4 2}$ & & \\
\hline JIF & $=\mathbf{1 . 5 0 0}$ & SJIF (Morocco) $=\mathbf{2 . 0 3 1}$ & & \\
\hline
\end{tabular}

\section{References:}

1. Gornushkin YG (1992) Practical advice owner of the car. - Moscow: The Patriot, 1992. - 207 p.

2. Ilyin IE (2012) The safety assessment of vehicle category // Transport safety and technology. - 2012. - № 6. - pp.13-17.

3. Krivchuk AS (2011) Problems of improvement of road safety // Transport Law. - 2011. - № 4. pp.12-19.

4. Kuznetsov AP (2015) Topical issues of road safety at the present stage // Transport Law. 2015. - № 1. - pp.19-25.

5. Mayboroda OV (2014) Fundamentals of driving and traffic safety: the textbook. - Moscow: Academy, 2014. - 256 p.
6. Ryabchinsky AI (2011) Regulation of active and passive safety of vehicles: Textbooks. Moscow: Academy, 2011. - 432 p.

7. Trofimov S (2012) Motor Vehicle Safety: Scientific and legal aspect // Lawyer. - 2012. № 8. - pp.12-18.

8. Yahyaev NY (2011) Security vehicles: a textbook. - Moscow: Academy, 2011. - 432 p.

9. (2015) The Ministry of Internal Affairs of the Russian Federation [the Electronic resource] Available: $\quad \underline{w w w . m v d . r u}$ (Accessed: 20.12.2015).

10. (2015) The traffic police of Russia [electronic resource] - Available: www.gibdd.ru (Accessed: 20.12.2015). 